\title{
NONPARAMETRIC ESTIMATION OF THE SINGULARITIES OF A SIGNAL FROM NOISY MEASUREMENTS
}

\author{
A. I. KATSEVICH AND A. G. RAMM
}

(Communicated by Wei Y. Loh)

\begin{abstract}
We study a problem of locating and estimating singularities of a signal measured with noise on a discrete set of points (fixed-design model). The signal consists of a smooth part with bounded first derivative and of finite number of singularities of the type $\left(x-t_{i}\right)_{ \pm}^{p} d_{i}, 0 \leq p \leq \frac{1}{2}$. The case $p=0$ corresponds to a piecewise continuous function. The algorithm is based on convolving the data with a kernel having compact support. Optimal bandwidth of the kernel is calculated, the consistency of the algorithm is proved. The results of testing the proposed algorithm on model examples are presented.
\end{abstract}

\section{INTRODUCTION}

The problem of locating singular points of a function is of interest in many applications [1-9]. For example, the recovery of discontinuity curves of a function $f(x), x \in \mathbb{R}^{2}$, from its Radon transform $\hat{f}(\alpha, t), 0 \leq \alpha \leq \pi, p \in \mathbb{R}$, leads to the problem of finding the singular locus of $\hat{f}(\alpha, t)[6,8]$, such that in a neighborhood of the point $\left(\alpha, t_{0}^{(i)}(\alpha)\right)$

$$
\hat{f}(\alpha, t)=s(\alpha, t)+d(\alpha)\left(t-t_{0}^{(i)}(\alpha)\right)_{ \pm}^{p}, \quad p=\frac{1}{2}, d(\alpha) \neq 0,
$$

where $s(\alpha, t)$ is a smooth function, and

$$
\left(x-t_{0}\right)_{ \pm}^{p}= \begin{cases}\left|x-t_{0}\right|^{p} & \text { if } \pm\left(x-t_{0}\right) \geq 0, \\ 0 & \text { if } \pm\left(x-t_{0}\right) \leq 0 .\end{cases}
$$

In this paper we develop an algorithm for locating and estimating singularities of a signal $s(x)$ measured with noise at a discrete equidistant set of points. The signal is supposed to have a finite number of singularities of the type (1.1) with known exponent $p, 0 \leq p \leq \frac{1}{2}$. The idea of the algorithm resembles that of the nonparametric kernel estimation [4] and consists in convolving the data with an odd piecewise constant kernel having compact support.

Similar problems have been studied in $[2,3]$ using global approach based on modified smoothing splines. In the particular case $p=0$ our algorithm reduces

Received by the editors November 6, 1992.

1991 Mathematics Subject Classification. Primary 62G05.

Key words and phrases. Noisy data, singularity localization, kernel estimation.

The authors thank ONR for support and Professor G. Wahba for pointing out several references. 
to the template matching technique which is used within a different framework in computer vision for edge detection $[1,5]$.

The paper is organized as follows. In $\S 2$ we describe the algorithm and calculate the optimal kernel bandwidth. Section 3 is devoted to the investigation of basic properties of the proposed algorithm. We show that this algorithm is consistent in the following sense: for any a priori fixed probability $\epsilon, 0<\epsilon<1$, of the first kind error, "there is a singularity at a given point when there is none" and for any "size" $d \neq 0$ of a singularity $d\left(x-x_{0}\right)_{ \pm}^{p}$ the probability of missing it and the accuracy of its localization go to zero as the stepsize goes to zero. In $\S 4$ we briefly discuss the case $p=0$ (localization and estimation of discontinuities of a piecewise differentiable signal) as a particular case of the general theory developed in $\S \S 2$ and 3 . The numerical results of testing the proposed algorithm are presented in $\S 5$. The proofs of all lemmas from $\S 3$ are given in the Appendix.

\section{FINDING SINGULARITIES OF A PIECEWISE-SMOOTH FUNCTION}

We use the following model. Let the known data be

$$
u_{i}=s_{i}+n_{i}, \quad s_{i}:=s\left(x_{i}\right), \quad x_{i}=i h, \quad i \in \mathbb{Z},
$$

where $\mathbb{Z}=\{0, \pm 1, \pm 2, \ldots\}$ and the random errors $n_{i}$ are independent, identically distributed with

$$
E\left(n_{i} n_{j}\right)=\left\{\begin{array}{ll}
\sigma^{2} & \text { if } i=j, \\
0 & \text { if } i \neq j,
\end{array} \quad E\left(n_{i}\right)=0 .\right.
$$

The signal is assumed to be of the form

$$
s(x)=\hat{s}(x)+\sum_{k}\left(x-t_{k}\right)_{ \pm}^{p} d_{k}, \quad d_{k}=\text { const }, 0 \leq p \leq \frac{1}{2}, \quad x \in \mathbb{R}^{1} .
$$

Here $\hat{s}(x)$ is a smooth part of the signal with the known estimate of its first derivative

$$
\left|\hat{s}^{\prime}(x)\right| \leq C \text {. }
$$

The points $t_{k}$ will be called singularities or singular points (that is, the points at which a one-sided limit of $s^{\prime}(x)$ equals infinity if $p>0$ or $s(x)$ has a jump if $p=0), d_{k}$ will be called coefficients of the singularities, and $p$ will be called the exponent of singularity. The singularities $\left(x-t_{k}\right)_{ \pm}^{p}$ will be called right and left respectively, or "+" and "-" type singularities. We assume that $t_{k}$ are located sufficiently far from each other. This restriction will be formulated precisely later. The problem is to find points $t_{k}$ and values $d_{k}$ and determine the type of singularity: if it is $\left(x-t_{k}\right)_{+}^{p}$ or $\left(x-t_{k}\right)_{-}^{p}$. First we derive an algorithm for the solution of this problem and then investigate its properties.

Let us assume that the singular point $t_{0}$ is at the middle of the interval $\left[x_{j}, x_{j+1}\right]: t_{0}=\left(x_{j}+x_{j+1}\right) / 2$, and consider the " + " type singularity

$$
\begin{cases}s(x)=\hat{s}(x)+\left(x-t_{0}\right)^{p} d_{0}, & x \geq t_{0}, \quad 0 \leq p \leq \frac{1}{2} . \\ s(x)=\hat{s}(x), & x<t_{0},\end{cases}
$$

We do not include other singularities $(k \neq 0)$ in $(2.4)$, since we assume that they are located outside the neighborhood $\Delta_{0}$ of $t_{0}$ in which we work. In 
particular, it is assumed that

$$
\left|\left(\hat{s}(x)+\sum_{k \neq 0}\left(x-t_{k}\right)_{ \pm}^{p} d_{k}\right)^{\prime}\right|=\left|\hat{s}^{\prime}(x)+\sum_{k \neq 0}\left( \pm \frac{p d_{k}}{\left(x-t_{k}\right)_{ \pm}^{1-p}}\right)\right| \leq C, \quad x \in \Delta_{0} \text {. }
$$

The last inequality holds if the distance between two neigboring singularities satisfies

$$
\left|t_{k+1}-t_{k}\right| \geq A\left(\frac{p d^{\max }}{C}\right)^{\frac{1}{1-p}},
$$

where $A$ is a sufficiently large number and $d^{\max }$ is the upper bound of the singularity coefficients. Similarly, it is assumed that in a neighborhood of each singularity the derivative of a smooth part of a signal still has the bound $C$. Let us define the following estimate $\tilde{d}_{j}$ of $d_{0}$ :

$$
\tilde{d}_{j}=\frac{\sum_{i=1}^{M} u_{i+j}-\sum_{i=0}^{M-1} u_{-i+j}}{h^{p} \sum_{i=1}^{M}\left(i-\frac{1}{2}\right)^{p}} .
$$

Representing the signal on both sides of $t_{0}$ by the Lagrange formula, one has

$$
\begin{aligned}
& s_{i+j}=\hat{s}\left(t_{0}\right)+\left(i-\frac{1}{2}\right) h \hat{s}^{\prime}\left(\xi_{i}\right)+\left(\left(i-\frac{1}{2}\right) h\right)^{p} d_{0}, \quad \xi_{i} \in\left(t_{0}, t_{0}+\left(i-\frac{1}{2}\right) h\right), \\
& s_{-i+j}=\hat{s}\left(t_{0}\right)-\left(i+\frac{1}{2}\right) h \hat{s}^{\prime}\left(\xi_{-i}\right), \quad \xi_{-i} \in\left(t_{0}-\left(i+\frac{1}{2}\right) h, t_{0}\right) .
\end{aligned}
$$

Substituting (2.1) and (2.7) into (2.6) and using (2.3), one obtains a formula for the error of the estimate (2.6):

$$
\begin{aligned}
\left|\tilde{d}_{j}-d_{0}\right| & =\frac{\left|\sum_{i=1}^{M} h\left(i-\frac{1}{2}\right)\left[\hat{s}^{\prime}\left(\xi_{i}\right)+\hat{s}^{\prime}\left(\xi_{-i+1}\right)\right]+\sum_{i=1}^{M}\left(n_{j+i}-n_{j-i+1}\right)\right|}{h^{p} \sum_{i=1}^{M}\left(i-\frac{1}{2}\right)^{p}} \\
& \leq \frac{2 C h \sum_{i=1}^{M}\left(i-\frac{1}{2}\right)+\left|\sum_{i=1}^{M}\left(n_{j+i}-n_{j-i+1}\right)\right|}{h^{p} \sum_{i=1}^{M}\left(i-\frac{1}{2}\right)^{p}}=\frac{C M^{2} h+|\nu|}{h^{p} \sum_{i=1}^{M}\left(i-\frac{1}{2}\right)^{p}},
\end{aligned}
$$

where

$$
\nu=\sum_{i=1}^{M}\left(n_{j+i}-n_{j-i+1}\right) .
$$

Let us fix $\epsilon, 0<\epsilon<1$, and find $x_{\epsilon}$ such that $P\left\{|\nu|>x_{\epsilon}\right\}=\epsilon$, where $P$ stands for probability. Assuming that $M$ is sufficiently large and using the Central Limit Theorem (or assuming that $n_{i}$ are Gaussian), one gets from (2.2)

$$
\nu \in \mathcal{N}\left(0,2 M \sigma^{2}\right),
$$

where $\mathscr{N}\left(0, \sigma^{2}\right)$ is the class of the Gaussian random variables with zero mean value and variance $\sigma^{2}$. Thus $x_{\epsilon}$ satisfies the equation $\operatorname{erfc}\left(x_{\epsilon} / 2 \sqrt{M} \sigma\right)=\epsilon$, where $\operatorname{erfc}(x)=\frac{2}{\sqrt{\pi}} \int_{x}^{\infty} \exp \left(-t^{2}\right) d t$. Hence, $x_{\epsilon}=2 \sqrt{M} \sigma \operatorname{erfc}^{-1}(\epsilon)$. From (2.8) it follows that the inequality

$$
\left|\tilde{d}_{j}-d_{0}\right| \leq \frac{C M^{2} h+2 \sqrt{M} \sigma \operatorname{erfc}^{-1}(\epsilon)}{h^{p} \sum_{i=1}^{M}\left(i-\frac{1}{2}\right)^{p}}:=\Delta d(M)
$$


holds with probability $1-\epsilon$. Minimizing the right-hand side of $(2.11)$ with respect to $M$ yields the optimal value $M^{\text {opt }}$ and the optimal value of the estimate $\Delta d^{\text {opt }}$. It is easy to see that $(2.11)$ holds also for the "-" type singularity $\left(x-t_{0}\right)_{-}^{p} d_{0}$. If there is no singularity in the interval $\Delta_{j}:=\left[x_{j-M^{\mathrm{opt}}+1}, x_{j+M^{\mathrm{opt}}}\right]$, the largest possible value of the estimate $(2.11)$ is also bounded by $\Delta d^{\text {opt }}$ with probability $1-\epsilon$ (take $d_{0}=0$ in $\left.(2.11)\right)$. Therefore, one can formulate the following

A. Algorithm for locating and estimating singularities of a signal. Consider the points $x_{k_{n}}$ at which $\left|\tilde{d}_{k_{n}}\right|>\Delta d^{\text {opt }}$, and assume that these points are arranged so that $\left|\tilde{d}_{k_{1}}\right|>\left|\tilde{d}_{k_{2}}\right|>\cdots$. The point $\tilde{x}_{1}:=\left(x_{k_{1}}+x_{k_{1}+1}\right) / 2$ is taken as the estimate of the singular point. The corresponding value $\tilde{d}_{k_{1}}$ is taken as the estimate of the coefficient of the singularity. Delete all the points from the set $\left\{x_{k_{n}}\right\}$ such that $\left|x_{k_{n}}-\tilde{x}_{1}\right|<L$, where $L=\max \left(M^{\text {opt }} h, A\left(p d^{\max } / C\right)^{\frac{1}{1-p}}\right)$ and $A$ is the constant in (2.5). Let $x_{k_{j}}$ be the point with the largest value of $\left|\tilde{d}_{k_{j}}\right|$ among the remaining ones. Then $\tilde{x}_{2}:=\left(x_{k_{j}}+x_{k_{j}+1}\right) / 2$ and $\tilde{d}_{k_{j}}$ are taken as the estimates of the next singular point and the coefficient of the singularity, respectively. Repeating this process, one estimates all singular points and corresponding coefficients.

If the conclusion has been made that there is a singularity at the middle of the current interval, we need to determine if it is of "+" or "-" type. To this end let us calculate the variation of the observed data for the left and right parts of the interval $\Delta_{j}$ separately. Since the derivative of the signal in the neighborhood of singularity is large compared to $C$, the variance of the signal will be large too, and one can conclude that the side with larger variance corresponds to the side with singularity. Note that this does not hold if $p=0$, i.e., if the signal has discontinuity. But in this case one needs only to determine the sign of a jump which is given by the sign of the estimate (2.6). Let us also note that the exponents $p$ of the singularities can vary from a singular point to another singular point. However, one has to assume that these exponents are known.

\section{INVESTIGATION OF THE BASIC PROPERTIES OF THE ALGORITHM}

First let us calculate the probabilities of errors of each kind. From (2.8)(2.11) it follows that the probability of the conclusion "there is a singularity at a given point" when there is none is less than $\epsilon$. To find the probability $\delta$ of missing a singularity let us assume that the singularity is of the type $\left(x-t_{0}\right)_{+}^{p} d_{0}$ with $d_{0}>0$. Then one has from (2.3), (2.9), and (2.10)

$$
\begin{aligned}
\delta & =P\left\{\tilde{d}_{j}<\Delta d^{\mathrm{opt}}\right\} \\
& =P\left\{d_{0}+\frac{\sum_{i=1}^{M^{\mathrm{opt}}}\left(i-\frac{1}{2}\right) h\left[\hat{s}^{\prime}\left(\xi_{i}\right)+\hat{s}^{\prime}\left(\xi_{-i+1}\right)\right]+\nu}{h^{p} \sum_{i=1}^{M^{\mathrm{opt}}}\left(i-\frac{1}{2}\right)^{p}}<\Delta d^{\mathrm{opt}}\right\} \\
& \leq P\left\{-\nu>d_{0} h^{p} \sum_{i=1}^{M^{\mathrm{opt}}}\left(i-\frac{1}{2}\right)^{p}-2 C\left(M^{\mathrm{opt}}\right)^{2} h-2 \sqrt{M^{\mathrm{opt}}} \sigma \operatorname{erfc}^{-1}(\epsilon)\right\} \\
& =\frac{1}{2} \operatorname{erfc}\left(\frac{d_{0} h^{p} \sum_{i=1}^{M^{\mathrm{opt}}}\left(i-\frac{1}{2}\right)^{p}-2 C\left(M^{\mathrm{opt}}\right)^{2} h}{2 \sqrt{M^{\mathrm{opt}}} \sigma}-\operatorname{erfc}^{-1}(\epsilon)\right) .
\end{aligned}
$$


To study the asymptotics of $\delta$ as $h \rightarrow 0$, one needs the following lemma (the proofs of all lemmas from this section are given in the appendix):

Lemma 1. One has

$$
M^{\mathrm{opt}}=c_{1} h^{-2 / 3}(1+o(1)), \quad h^{p} \sum_{i=1}^{M^{\mathrm{opt}}}\left(i-\frac{1}{2}\right)^{p}=c_{2} h^{\frac{p-2}{3}}(1+o(1)) \quad \text { as } h \rightarrow 0,
$$

where the constants $c_{1}, c_{2}>0$ depend only on $C, \sigma, p$ and do not depend on $h$.

Substituting (3.2) into (3.1), one gets

$$
\begin{aligned}
\delta & \leq \frac{1}{2} \operatorname{erfc}\left(\frac{d_{0} c_{2} h^{\frac{p-2}{3}}(1+o(1))-2 C c_{1}^{2}(1+o(1))^{2} h^{-4 / 3} h}{2 \sqrt{c_{1} h^{-2 / 3}(1+o(1))} \sigma}-\operatorname{erfc}^{-1}(\epsilon)\right) \\
& =\frac{1}{2} \operatorname{erfc}\left(c_{3} h^{\frac{p-1}{3}}-c_{4}\right) \leq \frac{1}{\sqrt{\pi}} \frac{\exp \left\{-\left(c_{3} h^{\frac{p-1}{3}}-c_{4}\right)^{2}\right\}}{\left(c_{3} h^{\frac{p-1}{3}}-c_{4}\right)} \rightarrow 0 \quad \text { as } h \rightarrow 0,
\end{aligned}
$$

since $p<1$. Here $c_{3}$ and $c_{4}$ are some constants and $\epsilon, 0<\epsilon<1$, is fixed. Clearly, the same result holds for $d_{0}<0$. Hence we proved the following

Theorem 1. For any fixed $\epsilon, 0<\epsilon<1$, and $d_{0} \neq 0$, the probability of missing singularity goes to zero as $h \rightarrow 0$.

To find the localization accuracy, let us assume that the singularity is of the form $\left(x-t_{0}\right)_{+}^{p} d_{0}$ with $d_{0}>0$ and let us compare the values $\tilde{d}_{j}$ and $\tilde{d}_{j+N}$, that is, consider the cases when the singularity is at the middle of the interval and when the interval has been shifted $N$ points to the right, $N \leq M^{\text {opt }}$. When the singularity is at the middle of the interval, $\tilde{d}_{j}$ is given by (2.6) with $M=M^{\text {opt }}$. When the interval has been shifted $N$ points to the right, the estimate is

$$
\tilde{d}_{j+N}=\frac{\sum_{i=1}^{M^{\mathrm{opt}}} u_{i+j+N}-\sum_{i=0}^{M^{\mathrm{opt}}-1} u_{-i+j+N}}{h^{p} \sum_{i=1}^{M^{\text {ol }}}\left(i-\frac{1}{2}\right)^{p}} .
$$

The localization accuracy is $N_{0} h$ if $\tilde{d}_{j}>\tilde{d}_{j+N}$ for $N_{0}<N \leq M^{\text {opt }}$. Hence, from (2.6) and (3.3) one gets

$$
\sum_{i=1}^{M^{\text {opt }}} u_{i+j}-\sum_{i=0}^{M^{\text {opt }}-1} u_{-i+j}>\sum_{i=1}^{M^{\text {opt }}} u_{i+j+N}-\sum_{i=0}^{M^{\text {opt }}-1} u_{-i+j+N}
$$

for all $N_{0}<N \leq M^{\text {opt }}$, where $N_{0}$ is to be determined. Canceling the similar terms on the left and right sides of (3.4) and using (2.1), (2.7), one obtains

$$
\begin{aligned}
& d_{0} h^{p}\left(2 \sum_{i=1}^{N}\left(i-\frac{1}{2}\right)^{p}-\sum_{i=1}^{N}\left(i+M^{\mathrm{opt}}-\frac{1}{2}\right)^{p}\right) \\
&>\left\{-\left(\sum_{i=1}^{N}\left(i-\frac{1}{2}\right) h \hat{s}^{\prime}\left(\xi_{i}\right)\right.\right.\left.+\sum_{i=M^{\mathrm{opt}}-N+1}^{M^{\mathrm{opt}}}\left(i-\frac{1}{2}\right) h \hat{s}^{\prime}\left(\xi_{-i+1}\right)\right) \\
&\left.+M^{\mathrm{opt}} h \sum_{i=1}^{N} \hat{s}^{\prime}\left(\eta_{i}\right)\right\}+\tilde{\nu}, \quad \eta_{i} \in\left(x_{i+j} x_{i+j+M^{\mathrm{opt}}}\right),
\end{aligned}
$$


where

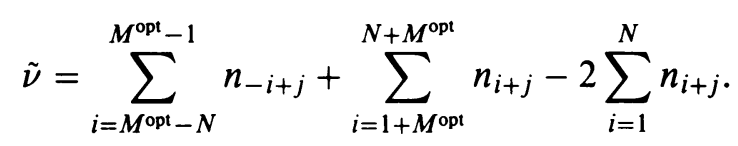

The term in the braces on the right-hand side of (3.5) can be estimated as follows:

$$
\begin{aligned}
& \left|-\left(\sum_{i=1}^{N}\left(i-\frac{1}{2}\right) h \hat{s}^{\prime}\left(\xi_{i}\right)+\sum_{i=M^{\mathrm{opt}}-N+1}^{M^{\mathrm{opt}}}\left(i-\frac{1}{2}\right) h \hat{s}^{\prime}\left(\xi_{-i+1}\right)\right)+M^{\mathrm{opt}} h \sum_{i=1}^{N} \hat{s}^{\prime}\left(\eta_{i}\right)\right| \\
& \leq C h\left(\frac{N(N+1)}{2}-\frac{N}{2}+\frac{M^{\mathrm{opt}}\left(M^{\mathrm{opt}}+1\right)}{2}\right. \\
& \left.\quad-\frac{\left(M^{\mathrm{opt}}-N\right)\left(M^{\mathrm{opt}}-N+1\right)}{2}-\frac{N}{2}+M^{\mathrm{opt}} N\right) \\
& =2 C M^{\mathrm{opt}} N h .
\end{aligned}
$$

Let us fix $\epsilon^{\prime}, 0<\epsilon^{\prime}<1$, and find $x_{\epsilon^{\prime}}$ such that

$$
P\left\{\tilde{\nu}>x_{\epsilon^{\prime}}\right\}=\epsilon^{\prime} \text {. }
$$

Let us assume that $n_{i}$ are Gaussian. Since the linear combination of Gaussian random variables is Gaussian, one gets from (2.2) and (3.6)

$$
E(\tilde{\nu})=0, \quad \sigma^{2}(\tilde{\nu})=6 N \sigma^{2}, \quad \tilde{\nu} \in \mathcal{N}\left(0,6 N \sigma^{2}\right) .
$$

Hence

$$
x_{\epsilon^{\prime}}=\sqrt{12 N} \sigma \operatorname{erfc}^{-1}\left(2 \epsilon^{\prime}\right) .
$$

Substitution of (3.7) and (3.10) into (3.5) yields

$$
d_{0} h^{p} f_{p}\left(N, M^{\mathrm{opt}}\right)>2 C M^{\mathrm{opt}} h N+\sqrt{12 N} \sigma \operatorname{erfc}^{-1}\left(2 \epsilon^{\prime}\right), \quad N_{0}<N \leq M^{\mathrm{opt}},
$$

where

$$
f_{p}(N, M)=2 \sum_{i=1}^{N}\left(i-\frac{1}{2}\right)^{p}-\sum_{i=1}^{N}\left(i+M-\frac{1}{2}\right)^{p} .
$$

The smallest value $N_{0}$ such that (3.11) holds for all $N, N_{0}<N \leq M^{\text {opt }}$, guarantees the localization accuracy $N_{0} h$ with probability $1-\epsilon^{\prime}$. The existence of such $N_{0}$ for sufficiently large $d_{0}$ follows from the following lemma.

Lemma 2. If $0 \leq p \leq \frac{1}{2}$ and $M \geq 1$, then $f_{p}(M, M)>0$.

Therefore, if we need the accuracy $N_{0} h$, it will be guaranteed with probability $1-\epsilon^{\prime}$ if the following two conditions hold:

$$
\begin{gathered}
d_{0}>\max _{N_{0}+1 \leq N \leq M^{\text {opt }}} \frac{2 C M^{\mathrm{opt}} h N+\sqrt{12 N} \sigma \mathrm{erfc}^{-1}\left(2 \epsilon^{\prime}\right)}{h^{p} f_{p}\left(N, M^{\mathrm{opt}}\right)}, \\
f_{p}\left(N, M^{\mathrm{opt}}\right)>0 \text { for } N_{0}+1 \leq N \leq M^{\mathrm{opt}} .
\end{gathered}
$$

From (3.12) it follows that $f_{p}(N, M)$ as a function of $N$ is increasing for

$$
N>y_{p}(M):=\frac{1}{2}+\frac{M}{2^{1 / p}-1} \geq \frac{M}{3}+\frac{1}{2} \text {. }
$$


Therefore, if $f_{p}\left(N_{0}, M^{\text {opt }}\right)>0$ and $N_{0}>y_{p}\left(M^{\text {opt }}\right)$, then $f_{p}\left(N, M^{\text {opt }}\right)>0$ for all $N \geq N_{0}$. Note that inequality (3.13) by itself is not sufficient to guarantee the localization accuracy $N_{0} h$, because it may happen that the local maximum of the estimate (2.6) is situated to the left of singular point $t_{0}$ or farther than $M^{\text {opt }}$ points to the right of $t_{0}$. Hence, one needs Lemma 3, which asserts that this is not the case. Repeating calculations similar to (3.3)-(3.13), one obtains

Lemma 3. (a) The case when the interval has been shifted $N$ points to the right, $M^{\mathrm{opt}}+1 \leq N \leq 2 M^{\mathrm{opt}}$, leads to an inequality weaker than (3.13) with $N_{0}=$ $M^{\mathrm{opt}}-1, N=M^{\mathrm{opt}}$.

(b) The case when the interval has been shifted $N$ points to the left, $1 \leq N \leq$ $M^{\mathrm{opt}}$, leads to an inequality weaker than (3.13) with the same value of $N$.

In fact, Lemma 3 means that the singularity $\left(x-t_{0}\right)_{ \pm}^{p} d_{0}$ with the exponent $0 \leq p \leq 1 / 2$ does not "propagate" too far: the maximum value of the estimate (2.6) is in the interval $\left[t_{0}-M^{\text {opt }} h, t_{0}+M^{\text {opt }} h\right]$ with the probability $1-\epsilon^{\prime}$, provided that $d_{0}$ is sufficiently large (see (3.13)). The reason for considering only the cases formulated in Lemma 3 is clear from (2.6): the estimate $\tilde{d}_{j}$ is proportional to the average value of the derivative of the signal in the interval $\Delta_{j}=\left[x_{j-M^{\mathrm{opt}+1}}, x_{j+M^{\mathrm{opt}}}\right]$. If one shifts $\Delta_{j}$ more than $M^{\text {opt }}$ points to the left, then $t_{0} \notin \Delta_{j}$ and the derivative of the signal is bounded by $C$. Hence the estimate (2.6) will exceed the threshold $\Delta d^{\text {opt }}$ with probability less than $\epsilon$. The situation is different if one shifts the interval to the right of $t_{0}$, since the derivative of the signal is large in the neighborhood of singularity. But, since the derivative of the singular term is decreasing as the distance from $t_{0}$ is increasing, one sees that there is no need to consider $N>2 M^{\text {opt }}$, because $\Delta_{j+2 M^{\text {opt }}}$ is the closest interval to $t_{0}$ such that $\Delta_{j+N} \cap \Delta_{j}=\varnothing$.

Let us now study the behavior of the right-hand side of (3.13a) as $h \rightarrow 0$. Consider the worst case when $N_{0}+1=M^{\mathrm{opt}}$. In this case (3.13a) reduces to

$$
d_{0}>d_{0}^{\min }(h):=\frac{2 C\left(M^{\mathrm{opt}}\right)^{2} h+\sqrt{12 M^{\mathrm{opt}}} \sigma \operatorname{erfc}^{-1}\left(2 \epsilon^{\prime}\right)}{h^{p} f_{p}\left(M^{\mathrm{opt}}, M^{\mathrm{opt}}\right)} .
$$

\section{Lemma 4.}

$$
d_{0}^{\min }(h)=c_{3} h^{\frac{1-p}{3}}(1+o(1)) \quad \text { as } h \rightarrow 0,
$$

where the constant $c_{3}$ does not depend on $h$.

The above lemmas yield the following result.

Theorem 2. For any $d_{0} \neq 0$ and accuracy $a>0$ of the localization of the singularity $\left(x-t_{0}\right)_{ \pm}^{p} d_{0}, 0 \leq p \leq \frac{1}{2}$, the probability of localization error larger than a goes to zero as $h \rightarrow 0$.

Proof. Suppose for simplicity that $d_{0}>0$. The case when $d_{0}<0$ can be considered similarly. Let us fix any $\epsilon^{\prime}>0$ and find $h_{0}$ such that the probability of an error larger than $a$ is less than $\epsilon^{\prime}$ for any $h \leq h_{0}$. Lemma 3 asserts that if one wants the accuracy $\left(M^{\text {opt }}-1\right) h$ with probability $1-\epsilon^{\prime}$, it is sufficient to satisfy (3.14). Using Lemmas 1 and 4, find $h_{1}$ and $h_{2}$ such that

$$
M^{\text {opt }} h_{1}=c_{1} h_{1}^{1 / 3}(1+o(1))<a, \quad d_{0}^{\min }\left(h_{2}\right)=c_{3} h_{2}^{\frac{1-p}{3}}(1+o(1))<d_{0} .
$$


Let $\Delta(h)$ be the localization error, and let $h_{0}=\min \left(h_{1}, h_{2}\right)$. Then, since $d_{0}^{\min }(h) \leq d_{0}^{\min }\left(h_{0}\right)<d_{0}$ and $M^{\text {opt }} h \leq M^{\text {opt }} h_{0}<a$, one has

$$
P\{\Delta(h)>a\}<P\left\{\Delta(h)>M^{\text {opt }} h\right\}<\epsilon^{\prime} \quad \text { for } h \leq h_{0} .
$$

Theorem 2 is proved.

\section{LOCALIZATION OF JUMPS}

Since the problem of jump localization from noisy data is practically important, we consider the case $p=0$ separately. Many formulas from previous sections can be considerably simplified if $p=0$. Letting $p=0$ in (2.11), one gets

$$
\left|\tilde{d}_{j}-d_{0}\right| \leq \Delta d(M)=C M h+\frac{2}{\sqrt{M}} \sigma \operatorname{erfc}^{-1}(\epsilon) .
$$

Minimization of the right-hand side of (4.1) with respect to $M$ yields

$$
\begin{aligned}
M^{\mathrm{opt}} & =\left[\left(\frac{\sigma \mathrm{erfc}^{-1}(\epsilon)}{C h}\right)^{2 / 3}\right], \\
\Delta d^{\mathrm{opt}} & =\Delta d\left(M^{\mathrm{opt}}\right) \approx 3\left(\operatorname{erfc}^{-1}(\epsilon)\right)^{2 / 3}(C h)^{1 / 3} \sigma^{2 / 3} .
\end{aligned}
$$

Note that (4.2) agrees with Lemma 1, which asserts $M^{\text {opt }}=O\left(h^{-2 / 3}\right)$ as $h \rightarrow 0$. Substitution of $p=0$ and (4.2) into (3.1) (with $d_{0}$ replaced by $\left|d_{0}\right|$ to cover cases $d_{0}>0$ and $d_{0}<0$ ) gives the probability of missing a jump given its value $d_{0}$ :

$$
\delta \leq \frac{1}{2} \operatorname{erfc}\left\{\frac{\left|d_{0}\right|}{2}\left(\frac{\operatorname{erfc}^{-1}(\epsilon)}{C h \sigma^{2}}\right)^{1 / 3}-2 \operatorname{erfc}^{-1}(\epsilon)\right\} .
$$

To find the accuracy of jump localization, put $p=0$ in (3.11) (with $d_{0}$ replaced by $\left.\left|d_{0}\right|\right)$ and (3.12). One has $f_{0}(N, M)=N$ and

$$
\left|d_{0}\right|>2 C M^{\mathrm{opt}} h+\sqrt{\frac{12}{N}} \sigma \operatorname{erfc}^{-1}\left(2 \epsilon^{\prime}\right), \quad N_{0}+1 \leq N \leq M^{\mathrm{opt}} .
$$

Since the right-hand side of (4.4) is a strictly decreasing function of $N$, one concludes that the localization accuracy $N_{0} h$ is guaranteed with probability $1-\epsilon^{\prime}$ if the jump magnitude $d_{0}$ satisfies (4.4) for $N=N_{0}+1$ (this inequality is much easier verifiable than the general inequality (3.13)). Conversely, the accuracy of the jump localization, given the jump magnitude $d_{0}$, is

$$
N_{0} h=\left[12\left(\frac{\sigma \operatorname{erfc}^{-1}\left(2 \epsilon^{\prime}\right)}{\left|d_{0}\right|-2 C M^{\mathrm{opt} h}}\right)^{2}\right] h .
$$

Define $N_{0}:=N_{0}\left(\epsilon^{\prime}\right)$ to be the expression in the brackets in equation (4.5). Let us summarize the results.

Theorem 3. Let $p=0$. Then the algorithm $A$ detects jumps with the probability $1-\delta$, where the estimate (4.3) of $\delta$ depends on the jump magnitude and on the fixed probability $\epsilon$ of the "false alarm" error. For any $\epsilon^{\prime}$ with $0<\epsilon^{\prime}<1$ and $N_{0}\left(\epsilon^{\prime}\right) \leq M^{\text {opt }}$, formula (4.5) gives the accuracy of the jump localization, such 
that the localization error larger than $N_{0} h$ occurs with the probability less than $\epsilon^{\prime}$.

\section{NUMERICAL EXPERIMENTS}

The algorithms described in $\S \S 2$ and 4 have been tested on model examples. Figure 1 shows the results of applying the algorithm for singularity localization. The test function (solid line)

$$
s(x)=0.02(x-5)^{2}+(x-5)_{+}^{0.3}
$$

is corrupted at each point by noise (dotted line) with uniform distribution, zero mean, and standard deviation $\sigma=0.15$. The observation points are $x_{i}=0.05 i, 0 \leq i \leq 200$. The probability of "false singularities" is chosen $\epsilon=$ 0.005 , the corresponding optimal bandwidth, obtained by minimizing $(2.11)$, is $M^{\text {opt }}=17$. The $x$-coordinate of the big dot represents the position of the found singularity $t_{0}=5.1$ (the true position is $t_{0}=5$ ). The $y$-coordinate of the dot is equal to the coefficient of the singularity estimated by (2.6) with $p=0.3$ : $d_{0}=1.04$ (the true value is $d_{0}=1$ ). No "false singularities" have been detected in this model. The probability of missing the singularity calculated by formula (3.1) is $\delta \leq 1.4 \cdot 10^{-9}$.

The results of testing the algorithm for jump localization are presented in Figure 2 on the next page. The test function (solid line)

$$
s(x)= \begin{cases}0.02(x-5)^{2}-0.5, & x<5, \\ 0.02(x-5)^{2}+0.5, & x>5\end{cases}
$$

is corrupted by the uniformly distributed noise (dotted line) with zero mean value and $\sigma=0.4$. The observation points are $x_{i}=\frac{1}{15} i, 0 \leq i \leq 150$. The probability $\epsilon$ is equal to 0.01 , and the corresponding $M^{\text {opt }}$, calculated by (4.2),

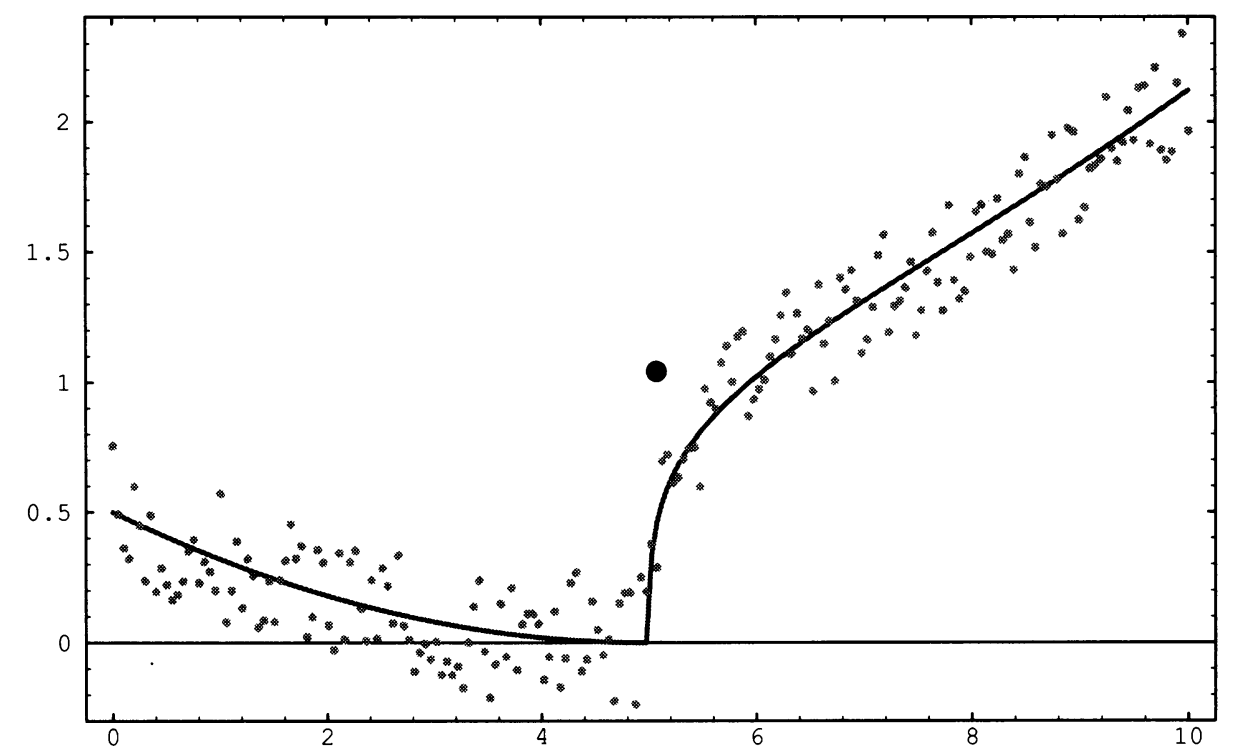

FIGURE 1. Singularity localization 


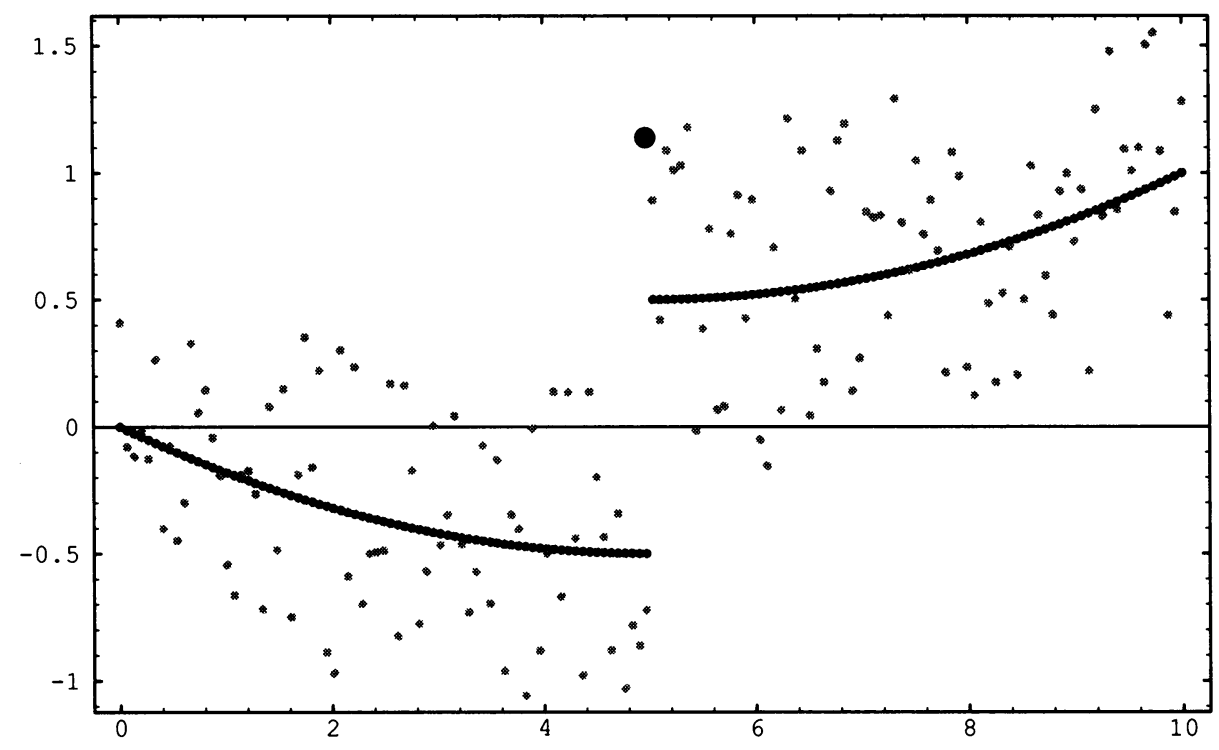

FIGURE 2. Jump localization

is 14 . The $x$-coordinate of the big dot represents the position of the estimated jump $t_{0}=5$ (which coincides with its true position), the $y$-coordinate of the dot equals the estimate of the jump magnitude $d_{0}=1.14$ (the true value is 1). No "false jumps" have been detected in this example. The probability of missing the jump calculated by formula (4.3) is $\delta \leq 6.1 \cdot 10^{-2}$.

\section{APPENDIX}

Proof of Lemma 1. Let us first prove the following simple formula

$$
\sum_{i=1}^{M}\left(i-\frac{1}{2}\right)=\frac{M^{p+1}}{p+1}(1+o(1)) \quad \text { as } M \rightarrow \infty .
$$

One has

$$
\int_{1}^{M}\left(x-\frac{1}{2}\right)^{p} d x<\sum_{i=1}^{M}\left(i-\frac{1}{2}\right)^{p}<\int_{1}^{M+1}\left(x-\frac{1}{2}\right)^{p} d x
$$

Thus

$$
\begin{aligned}
& \frac{\left|\sum_{i=1}^{M}\left(i-\frac{1}{2}\right)^{p}-\int_{0}^{M} x^{p} d x\right|}{\int_{0}^{M} x^{p} d x} \\
& \quad \leq \frac{\int_{M}^{M+1}\left(x-\frac{1}{2}\right)^{p} d x+\left(\int_{0}^{M} x^{p} d x-\int_{1}^{M}\left(x-\frac{1}{2}\right)^{p} d x\right)}{\int_{0}^{M} x^{p} d x} \\
& \quad=\frac{\left(M+\frac{1}{2}\right)^{p+1}-\left(M-\frac{1}{2}\right)^{p+1}+M^{p+1}-\left(M-\frac{1}{2}\right)^{p+1}+\left(\frac{1}{2}\right)^{p+1}}{M^{p+1}} \rightarrow 0
\end{aligned}
$$

as $M \rightarrow \infty$. Hence

$$
\sum_{i=1}^{M}\left(i-\frac{1}{2}\right)=\int_{0}^{M} x^{p} d x(1+o(1))=\frac{M^{p+1}}{p+1}(1+o(1)) \quad \text { as } M \rightarrow \infty .
$$


Now let us prove that $M^{\text {opt }} \rightarrow \infty$ as $h \rightarrow 0$. Let us argue by contradiction. Assume that there exists $M_{0}>0$ such that $M^{\text {opt }}<M_{0}$ as $h \rightarrow 0$. Pick any $\delta \ll 2 \sigma \operatorname{erfc}^{-1}(\epsilon)$, put $h_{0}=\delta / C M_{0}^{2}$, and fix any $h<h_{0}$ in (2.11). The numerator of (2.11) grows like $M^{1 / 2}$ (for $M \leq M_{0}$ ), and the denominator grows like $M^{p+1}$ (see (A.1)). Hence

$$
\Delta d\left(M_{0}+1\right)<\Delta d(M) \quad \forall M, 1 \leq M \leq M_{0} ;
$$

in particular, $\Delta d\left(M_{0}+1\right)<\Delta d\left(M^{\text {opt }}\right)$, which contradicts the definition of $M^{\text {opt }}$. To prove the first assertion of Lemma 1 , denote $t=M h$ and substitute (A.1) into (2.11) to get

$$
\begin{aligned}
\Delta d(t) & =\frac{C \frac{t^{2}}{h}+\sqrt{\frac{4 t}{h}} \sigma \operatorname{erfc}^{-1}(\epsilon)}{\frac{1}{h} \frac{t^{p+1}}{p+1}(1+o(1))} \\
& =(p+1)(1+o(1))\left[C t^{1-p}+\sqrt{4 h} t^{-1 / 2-p} \sigma \operatorname{erfc}^{-1}(\epsilon)\right] .
\end{aligned}
$$

The expression in brackets attains its minimum at $t=t^{\mathrm{opt}}$, where

$$
t^{\mathrm{opt}}=\left(\frac{2 \sigma \operatorname{erfc}^{-1}(\epsilon)(p+1 / 2)}{C(1-p)}\right)^{2 / 3} h^{1 / 3} .
$$

Thus $M^{\text {opt }}$ is given by the formula

$$
M^{\text {opt }}=c_{1} h^{-2 / 3}(1+o(1)),
$$

where

$$
c_{1}=\left(\frac{2 \sigma \operatorname{erfc}^{-1}(\epsilon)(p+1 / 2)}{C(1-p)}\right)^{2 / 3} .
$$

Substituting (A.3) into (A.2), one obtains

$$
\Delta d\left(M^{\mathrm{opt}}\right)=O\left(h^{\frac{1-p}{3}}\right) \quad \text { as } h \rightarrow 0 .
$$

Combining (A.1) and (A.3) yields the last assertion of Lemma 1.

Proof of Lemma 2. Let us consider first the case $p=\frac{1}{2}$.

$$
\begin{aligned}
f_{1 / 2}(M+1, M+1) & -f_{1 / 2}(M, M) \\
= & 2 \sqrt{M+1 / 2}-\sqrt{2 M+3 / 2} \\
& -\sum_{i=1}^{M}(\sqrt{i+M+1 / 2}-\sqrt{i+M-1 / 2}) \\
> & 2 \sqrt{M+1 / 2}-\sqrt{2 M+3 / 2}-\frac{1}{2} \sum_{i=1}^{M} \frac{1}{\sqrt{i+M-1 / 2}} \\
> & 2 \sqrt{M+1 / 2}-\sqrt{2 M+3 / 2}-\frac{1}{2} \int_{0}^{M} \frac{d x}{\sqrt{x+M-1 / 2}} \\
= & (2 \sqrt{M+1 / 2}+\sqrt{M-1 / 2})-(\sqrt{2 M+3 / 2}+\sqrt{2 M-1 / 2})>0 .
\end{aligned}
$$

One has $f_{1 / 2}(1,1)=(2-\sqrt{3}) / \sqrt{2}>0$, hence, $f_{1 / 2}(M, M)>0$ for all $M \geq 1$. Now let $0 \leq p \leq \frac{1}{2}$. One has

$$
f_{p}(M, M)=2 \sum_{i=1}^{M} \frac{\sqrt{i-1 / 2}}{(i-1 / 2)^{1 / 2-p}}-\sum_{i=1}^{M} \frac{\sqrt{i+M-1 / 2}}{(i+M-1 / 2)^{1 / 2-p}} .
$$


Since $\left(i-\frac{1}{2}\right)^{1 / 2-p}<M^{1 / 2-p}<\left(i+M-\frac{1}{2}\right)^{1 / 2-p}$ for $1 \leq i \leq M$, it follows that

$$
f_{p}(M, M)>\frac{1}{M^{1 / 2-p}} f_{1 / 2}(M, M)>0 .
$$

Proof of Lemma 3. Let us consider the first claim, $M^{\text {opt }}+1 \leq N \leq 2 M^{\text {opt }}$. The basic inequality is

$$
\sum_{i=1}^{M^{\mathrm{opt}}} u_{i+j}-\sum_{i=0}^{M^{\mathrm{opt}}-1} u_{-i+j}>\sum_{i=1}^{M^{\mathrm{opt}}} u_{i+j+N}-\sum_{i=0}^{M^{\mathrm{opt}}-1} u_{-i+j+N} .
$$

Canceling similar terms on the left and right sides of (A.4), using (2.1) and (2.4), one obtains

$$
\begin{aligned}
d_{0} h^{p} & \left(\sum_{i=1}^{M^{\mathrm{opt}}}\left(i-\frac{1}{2}\right)^{p}+\sum_{i=N-M^{\mathrm{opt}}+1}^{N}\left(i-\frac{1}{2}\right)^{p}-\sum_{i=N+1}^{M^{\mathrm{opt}}+N}\left(i-\frac{1}{2}\right)^{p}\right) \\
\geq & -\sum_{i=1}^{M^{\mathrm{opt}}}\left(i-\frac{1}{2}\right) h\left[\hat{s}^{\prime}\left(\xi_{i}\right)+\hat{s}^{\prime}\left(\xi_{-i+1}\right)\right]+M^{\mathrm{opt}} h \sum_{i=1}^{M^{\mathrm{opt}}} \hat{s}^{\prime}\left(\eta_{i}\right)+\tilde{\tilde{\nu}},
\end{aligned}
$$

where

$$
\text { (A.6) } \tilde{\tilde{\nu}}=\sum_{i=N+1}^{M^{\mathrm{opt}}+N} n_{j+i}+\sum_{i=0}^{M^{\mathrm{opt}}-1} n_{j-i}-\sum_{i=1}^{N-M^{\mathrm{opt}}} n_{j+i}-\sum_{i=1+M^{\mathrm{opt}}}^{N} n_{j+i}-2 \sum_{i=N-M^{\mathrm{opt}+1}}^{M^{\mathrm{opt}}} n_{j+i} \text {. }
$$

The last sum in (A.6) is defined to be zero if $N=2 M^{\text {opt }}$. Estimating the right-hand side of (A.5) with probability $1-\epsilon$, one gets

$$
d_{0} h^{p} F_{p}\left(N, M^{\mathrm{opt}}\right)>2 C\left(M^{\mathrm{opt}}\right)^{2} h+x_{\epsilon}, \quad M^{\mathrm{opt}}+1 \leq N \leq 2 M^{\mathrm{opt}},
$$

where

$$
F_{p}(N, M)=\sum_{i=1}^{M}\left(i-\frac{1}{2}\right)^{p}+\sum_{i=N-M+1}^{N}\left(i-\frac{1}{2}\right)^{p}-\sum_{i=N+1}^{M+N}\left(i-\frac{1}{2}\right)^{p}
$$

and $x_{\epsilon}$ is determined from the condition $P\left\{\tilde{\nu}>x_{\epsilon}\right\}=\epsilon$. From (A.6) and (2.2) it follows that $\tilde{\nu} \in \mathcal{N}\left(0,\left(8 M^{\text {opt }}-2 N\right) \sigma^{2}\right)$. Hence

$$
x_{\epsilon}=2 \sqrt{4 M^{\mathrm{opt}}-N} \sigma \operatorname{erfc}^{-1}(2 \epsilon),
$$

and (A.7) takes the form

$$
\begin{aligned}
d_{0} h^{p} F_{p}\left(N, M^{\mathrm{opt}}\right)>2 C\left(M^{\mathrm{opt}}\right)^{2} h+2 \sqrt{4 M^{\mathrm{opt}}-N} \sigma \mathrm{erfc}^{-1}(2 \epsilon), \\
M^{\mathrm{opt}}+1 \leq N \leq 2 M^{\mathrm{opt}} .
\end{aligned}
$$

Comparing (A.9) and (3.11) with $N=M^{\text {opt }}$, one sees that to prove the first assertion of Lemma 3 it is sufficient to show $F_{p}(N, M) \geq f_{p}(M, M)$ for $M+1 \leq N \leq 2 M$. From (3.12) and (A.8) it follows that one needs to prove the inequality

$$
2 \sum_{i=1}^{M}\left(i-\frac{1}{2}\right)^{p}-\sum_{i=M+1}^{2 M}\left(i-\frac{1}{2}\right)^{p} \leq \sum_{i=1}^{M}\left(i-\frac{1}{2}\right)^{p}+\sum_{i=N-M+1}^{N}\left(i-\frac{1}{2}\right)^{p}-\sum_{i=N+1}^{M+N}\left(i-\frac{1}{2}\right)^{p} .
$$


Cancelation of the similar terms yields the equivalent inequality

$$
\sum_{i=1}^{N-M}\left(i-\frac{1}{2}\right)^{p}+\sum_{i=2 M+1}^{M+N}\left(i-\frac{1}{2}\right)^{p} \leq 2 \sum_{i=M+1}^{N}\left(i-\frac{1}{2}\right)^{p},
$$

which can be rewritten as

$$
\text { (A.10) } \sum_{i=1}^{N-M}\left\{\left[\left(x_{i}+2 M\right)^{p}-\left(x_{i}+M\right)^{p}\right]-\left[\left(x_{i}+M\right)^{p}-x_{i}^{p}\right]\right\} \leq 0, \quad x_{i}=i-\frac{1}{2} \text {. }
$$

Since $0 \leq p \leq 1 / 2$ and $M>0$, one has

$$
\left(x_{i}+2 M\right)^{p}-\left(x_{i}+M\right)^{p}<\left(x_{i}+M\right)^{p}-x_{i}^{p},
$$

which proves (A.10) and the first claim in Lemma 3.

In a similar fashion it is easy to show that the case when the interval has been shifted $N$ points to the left, $1 \leq N \leq M^{\text {opt }}$, leads to an inequality weaker than (3.11) with the same $N$. Indeed, the resulting inequality is

$$
d_{0} h^{p} \sum_{i=M^{\mathrm{opt}}-N+1}^{M^{\mathrm{opt}}}\left(i-\frac{1}{2}\right)^{p}>2 C M^{\mathrm{opt}} h N+\sqrt{12 N} \sigma \operatorname{erfc}^{-1}(2 \epsilon), \quad 1 \leq N \leq M^{\mathrm{opt}},
$$

and from the equation

$$
\begin{aligned}
& \sum_{i=M-N+1}^{M}\left(i-\frac{1}{2}\right)^{p}-\left(2 \sum_{i=1}^{N}\left(i-\frac{1}{2}\right)^{p}-\sum_{i=1}^{N}\left(i+M-\frac{1}{2}\right)^{p}\right) \\
& =\sum_{i=1}^{N}\left(\left(i-\frac{1}{2}+M\right)^{p}+\left(i-\frac{1}{2}+M-N\right)^{p}-2\left(i-\frac{1}{2}\right)^{p}\right)>0
\end{aligned}
$$

one obtains the desired assertion.

Proof of Lemma 4. Using the approach from the proof of Lemma 1, one obtains the estimate

(A.11)

$$
h^{p} \sum_{i=1}^{M^{\mathrm{opt}}}\left(i+M^{\mathrm{opt}}-\frac{1}{2}\right)^{p}=\frac{\left(2 M^{\mathrm{opt}} h\right)^{p+1}-\left(M^{\mathrm{opt}} h\right)^{p+1}}{(p+1) h}(1+o(1)) \quad \text { as } h \rightarrow 0 .
$$

Combining (3.2), (3.12), (3.14), and (A.11) yields

$$
\begin{aligned}
d_{0}^{\min }(h) & =\frac{2 C\left(M^{\mathrm{opt}}\right)^{2} h+\sqrt{12 M^{\mathrm{opt}}} \sigma \operatorname{erfc}^{-1}\left(2 \epsilon^{\prime}\right)}{2 \frac{\left(M^{\mathrm{opt} h} h^{p+1}\right.}{(p+1) h}(1+o(1))-\frac{\left(2 M^{\mathrm{opt}} h\right)^{p+1}-\left(M^{\mathrm{op} h}\right)^{p+1}}{(p+1) h}(1+o(1))} \\
& =(p+1) \frac{2 C\left(M^{\mathrm{opt}} h\right)^{2}+\sqrt{12 M^{\mathrm{opt}}} h \sigma \mathrm{erfc}^{-1}\left(2 \epsilon^{\prime}\right)}{\left(3-2^{p+1}\right)\left(M^{\mathrm{opt}} h\right)^{p+1}(1+o(1))} \\
& =\frac{\text { const }}{3-2^{p+1}} \frac{h^{2 / 3}}{h^{(p+1) / 3}}(1+o(1))=\text { const }_{1} h^{\frac{1-p}{3}}(1+o(1)) \rightarrow 0 \text { as } h \rightarrow 0,
\end{aligned}
$$

where we used the inequality $2^{p+1}<3$ for $0 \leq p \leq \frac{1}{2}$. Lemma 4 is proved. 


\section{REFERENCES}

1. L. S. Davis, A survey of edge detection techniques, Comput. Graphics Image Processing 4 (1975), 248-270.

2. D. Girard, From template matching to optimal approximation by piecewise smooth curves, Curves and Surfaces in Computer Vision and Graphics, vol. 1251, The Society of PhotoOptical Instrumentation Engineers, Washington, DC, 1990, pp. 174-182.

3. D. Girard and P. J. Laurent, Splines and estimation of nonlinear parameters, Mathematical Methods in Computer Aided Geometric Design (T. Lyche and L. L. Schumaker, eds.), Academic Press, New York, 1989, pp. 273-298.

4. W. Härdle, Applied nonparametric regression, Cambridge Univ. Press, Cambridge, 1990.

5. W. K. Pratt, Digital image processing, 2nd ed., Wiley-Interscience, New York, 1991.

6. A. G. Ramm, Multidimensional inverse scattering problems, Longman, Wiley, New York, 1992 (expanded Russian edition, Mir, Moscow, 1993).

7. __ Random fields estimation theory, Longman, Wiley, New York, 1990.

8. A. G. Ramm and A. I. Zaslavsky, Reconstructing singularities of a function from its Radon transform, Bull. Amer. Math. Soc. (N.S.) 25 (1993), 109-115.

9. G. Wahba, Spline models for observational data, CBMS-NSF Regional Conf. Ser. Appl. Math., vol. 59, SIAM, Philadelphia, PA, 1990.

Department of Mathematics, Kansas State University, Manhattan, Kansas 665062602

E-mail address: katsev@ksuvm.ksu.edu

E-mail address: ramm@ksuvm.ksu.edu 